\title{
Long noncoding RNA UCA1 facilitates cell proliferation and inhibits apoptosis in retinoblastoma by activating the PI3K/Akt pathway
}

\author{
Zhongfang Yuan ${ }^{1}$, Zhaona $\mathrm{Li}^{2}$ \\ ${ }^{1}$ Department of Ophthalmology, Jinan Central Hospital Affiliated to Shandong University, Jinan 250013, China; ${ }^{2}$ Department of Ophthalmology, \\ The Second People's Hospital of Jinan, Jinan 250001, China \\ Contributions: (I) Conception and design: Z Yuan; (II) Administrative support: Z Yuan; (III) Provision of study materials or patients: Z Li; \\ (IV) Collection and assembly of data: Z Yuan; (V) Data analysis and interpretation: Z Yuan; (VI) Manuscript writing: All authors; (VII) Final approval \\ of manuscript: All authors. \\ Correspondence to: Zhaona Li, DM. Department of Ophthalmology, The Second People's Hospital of Jinan, No, 148, Jingyi Road, Jinan 250001, \\ China. Email: lizhaona133@163.com.
}

Background: This study aimed to explore the effects of the long noncoding RNA (lncRNA)-UCA1 on
retinoblastoma (RB) and the potential underlying molecular mechanisms.
Methods: The expression of lncRNA-UCA1 was measured by qRT-RCR in both RB tissues and the
RB cell lines HXO-RB44 and Y79. The relationship between lncRNA-UCA1 expression and the clinical
characteristics of RB patients was evaluated. Cell proliferation, colony formation, and apoptosis and the cell
cycle of HXO-RB44 and Y79 cells were evaluated by the cell counting kit-8 (CCK-8) assay, colony formation
assay, and flow cytometry, respectively. In addition, the expression levels of PCNA, caspase-3, survivin, p16,
p21, CDK2, PI3K, p-PI3K, Akt, p-Akt, and S6k in HXO-RB44 and Y79 cells were measured by Western
blotting.

Results: LncRNA-UCA1 was highly expressed in both RB tissues and the RB cell lines HXO-RB44 and Y79. Moreover, lncRNA-UCA1 expression levels in RB patients were correlated with tumour size, optic nerve invasion, and pathologic grade. LncRNA-UCA1 promoted cell proliferation and cell cycle progression and inhibited apoptosis in HXO-RB44 and Y79 cells. LncRNA-UCA1 overexpression dramatically increased the expression of S6k and the phosphorylation of PI3K and Akt in RB cells. Treatment with the PI3K inhibitor LY294002 reversed the effects of lncRNA-UCA1 on RB cell proliferation, apoptosis, and cell cycle progression.

Conclusions: Our study showed that lncRNA-UCA1 could promote cell proliferation and cell cycle progression and inhibit cell apoptosis in RB by activating the PI3K/Akt pathway.

Keywords: lncRNA-UCA1; retinoblastoma (RB); proliferation; apoptosis; PI3K/Akt pathway

Submitted Sep 04, 2019. Accepted for publication Nov 22, 2019.

doi: $10.21037 /$ tcr.2019.12.47

View this article at: http://dx.doi.org/10.21037/tcr.2019.12.47

\section{Introduction}

Retinoblastoma (RB) is the most common intraocular cancer, and it mainly affects children under 5 years of age $(1,2)$. The incidence of $\mathrm{RB}$ decreases with age, and it is higher among boys than among girls (3). Despite improvements in treatment, the survival rate of $\mathrm{RB}$ patients remains poor, mainly because of resistant to chemotherapy and radiotherapy (4). Therefore, research aimed at identifying new diagnostic markers and therapeutic targets for $\mathrm{RB}$ is urgently needed.

Long noncoding RNAs (lncRNAs) are defined as RNA transcripts longer than 200 nucleotides that have 
Table 1 Association between lncRNA-UCA1 expression and clinical parameters in RB patients

\begin{tabular}{|c|c|c|c|}
\hline Clinical parameters & $\mathrm{N}$ & UCA1 expression & $P$ value \\
\hline$\leq 2$ & 22 & $2.405 \pm 0.268$ & \\
\hline$>2$ & 20 & $2.416 \pm 0.300$ & \\
\hline Gender & & & 0.8395 \\
\hline Female & 22 & $2.408 \pm 0.299$ & \\
\hline Tumor size & & & $0.0074^{\star \star}$ \\
\hline$\leq 10 \mathrm{~mm}$ & 22 & $2.624 \pm 0.100$ & \\
\hline$>10 \mathrm{~mm}$ & 20 & $2.218 \pm 0.105$ & \\
\hline No & 17 & $2.133 \pm 0.108$ & \\
\hline Pathologic grade & & & $0.0078^{\star *}$ \\
\hline Poorly differentiated & 20 & $2.213 \pm 0.101$ & \\
\hline Well differentiated & 22 & $2.619 \pm 0.102$ & \\
\hline
\end{tabular}

**, $\mathrm{P}<0.01$.

no protein-coding ability (5). Accumulating evidence has shown that IncRNAs play important regulatory roles in the development and progression of various cancers, including RB (6). Studies have also confirmed that lncRNAs participate in numerous cellular activities, such as cell proliferation, migration, invasion, and cell cycle arrest $(7,8)$. For example, lncRNA-BDNF-AS has been reported to suppress the proliferation, migration, and cell cycle transition of RB cells (9). LncRNA-SNHG16 promoted cell proliferation and colony formation and inhibited apoptosis in RB (10). Dong et al. (11) confirmed that lncRNA-HOTAIR promoted cell proliferation and invasion by regulating the Notch pathway in RB. It was reported that lncRNA-H19 inhibited RB cell proliferation, migration, and invasion and promoted apoptosis (12). However, the effect of lncRNA-UCA1 in RB is not fully understood.

The phosphatidylinositol 3-kinase/protein kinase B (PI3K/Akt) pathway has been reported to play a crucial role in a variety of malignancies (13-15), and some lncRNAs exert their cancer-related effects by regulating the PI3K/Akt pathway (5). Previous studies have confirmed that lncRNAUCA1 promotes osteosarcoma metastasis by activating the PI3K/Akt pathway (16). Li et al. (17) reported that IncRNA-
UCA1 facilitates cell proliferation and suppresses apoptosis by regulating the PI3K/Akt pathway in gastric cancer. Therefore, we investigated whether lncRNA-UCA1 affects RB by regulating the PI3K/Akt pathway.

In this study, we explored the effects of IncRNAUCA1 on cell proliferation and apoptosis in RB and the related molecular mechanisms. Our results showed that lncRNA-UCA1 could facilitate cell proliferation and inhibit apoptosis in RB through activation of the PI3K/Akt pathway. Our results indicate that IncRNA-UCA1 may be useful as a new therapeutic target in RB.

\section{Methods}

\section{Clinical samples}

A total of 42 fresh RB specimens and paired adjacent normal retinal tissues from patients with $\mathrm{RB}$ were provided by the Ophthalmology Department of our hospital. None of the patients received anti-tumour treatment, chemotherapy, or radiotherapy before surgery. The characteristics of the study patients are presented in Table 1. The study was approved by the Ethics Committee of our hospital. All patients or their guardians provided written informed consent. 


\section{Cell cultures}

Two RB cell lines (HXO-RB44 and Y79) and a normal retinal vascular endothelial cell line (ACBRI-181) were obtained from American Type Culture Collection (ATCC, USA). All cells were cultured in RPMI-1640 medium (Gibco, USA) containing 10\% FBS and 1\% penicillin/streptomycin in a humidified incubator with $5 \% \mathrm{CO}_{2}$ at $37^{\circ} \mathrm{C}$.

\section{Cell transfection assay}

PcDNA3.1-UCA1, UCA1 siRNA, and the corresponding negative controls (pcDNA3.1-Mock and UCA1 siRNA control, respectively) were obtained from Thermo Fisher (USA). LY294002 (a PI3K inhibitor) was purchased from Sigma (USA). pcDNA3.1-UCA1, pcDNA3.1Mock, UCA1 siRNA, and UCA1 siRNA control were separately transfected into HXO-RB44 and Y79 cells using Lipofectamine ${ }^{\circledR} 3000$ Reagent (Invitrogen, USA). Transfected HXO-RB44 and Y79 cells were randomly divided into six groups that were treated as follows: BLANK group (non-treated), pcDNA-UCA1 group (transfected with pcDNA3.1-UCA1), pcDNA-Mock group (transfected with pcDNA3.1-Mock), si-UCA1 group (transfected with UCA1 siRNA), si-NC group (transfected with UCA1 siRNA control), and pcDNA-UCA1 + LY294002 group (transfected with pcDNA-UCA1 and treated with $50 \mu$ M LY294002). All transfected cells were cultured at $37^{\circ} \mathrm{C}$ for $48 \mathrm{~h}$.

\section{Cell counting kit-8 (CCK-8) assay}

The cell proliferation abilities of HXO-RB44 and Y79 cells were assessed by the CCK-8 kit (Sigma). In brief, transfected HXO-RB44 and Y79 cells were seeded into 96-well plates (at $1 \times 10^{4}$ cells/well). At different time points $(24,48,72$, and $96 \mathrm{~h}), 10 \mu \mathrm{L}$ of CCK-8 reagent was added to each well and the cells were incubated at room temperature for $1 \mathrm{~h}$. Finally, the absorbance was measured at $450 \mathrm{~nm}$ with a microplate reader (Bio-Rad, USA).

\section{Colony formation assay}

The transfected HXO-RB44 and Y79 cells were respectively seeded into 6-well plates at a density of 300 cells per well and cultured for $14 \mathrm{~d}$. After fixation with $4 \%$ cold formaldehyde for $30 \mathrm{~min}$, the colonies were stained with $0.1 \%$ crystal violet (Beyotime, China) for $20 \mathrm{~min}$. Finally, the colonies were observed, photographed, and counted under an optical microscope (Olympus, Tokyo, Japan).

\section{Cell cycle analyses}

Transfected HXO-RB44 and Y79 cells were harvested, washed with PBS, and fixed in pre-chilled $70 \%(\mathrm{v} / \mathrm{v})$ ethanol for $2 \mathrm{~h}$ at $-20^{\circ} \mathrm{C}$. Then, the cells were stained with Muse ${ }^{\mathrm{TM}}$ Cell Cycle Reagent (Merck Millipore, USA) for $30 \mathrm{~min}$ in the dark. Finally, the cells were observed and analysed with a MUSE ${ }^{\mathrm{TM}}$ flow cytometer (Merck Millipore, USA).

\section{Apoptosis assay}

Apoptosis of HXO-RB44 and Y79 cells was assessed by using the Annexin V-FITC apoptosis detection kit (Invitrogen, USA). In brief, transfected HXO-RB44 and Y79 cells were collected and resuspended in binding buffer and then stained with Annexin V-FITC and propidium iodide (PI) for $15 \mathrm{~min}$ in the dark. Finally, apoptotic cells were observed with a MUSE ${ }^{\mathrm{TM}}$ flow cytometer.

\section{Real-time fluorogenic PCR assays}

Total RNA from RB samples, adjacent normal retinal tissues, and HXO-RB44, Y79, and ACBRI-181 cells was extracted using TRIZOL reagent (Invitrogen, USA). Then, cDNA was synthesized from the extracted total RNA using the Revert Aid First Strand cDNA Synthesis Kit (Thermo, USA). Next, qRT-PCR was performed using SYBR Green qPCR Master Mix (Thermo Scientific, USA) according to the manufacturer's protocol. The primers used in this experiment were as follows: lncRNAUCA1 F, 5'-CCACACCCAAAACAAAAAATCT-3' and R, 5'-TCCCAAGCCCTCTAACAACAA-3'; GAPDH F, 5'-TGTTCGTCATGGGTGTGAAC-3' and R, 5'-ATGGCATGGACTGTGGTCAT-3'.

\section{Western blot analysis}

Total proteins were extracted with RIPA buffer (Beyotime, Jiangsu, China). Then, samples containing $50 \mu \mathrm{g}$ of protein were separated by $10 \%$ SDS-PAGE and then transferred to nitrocellulose membranes. The membranes were blocked with $5 \%$ milk in TBST for $2 \mathrm{~h}$, and then incubated overnight at $4{ }^{\circ} \mathrm{C}$ with the following primary antibodies [PI3K, 1:1,000; Akt, 1:1,000; PCNA, 1:1,000; and GAPDH, 1:1,000 (all from Sino Biological, USA); p-PI3K, 1:500; p-Akt, 1:500; and S6K, 1:1,000 (all from 
A

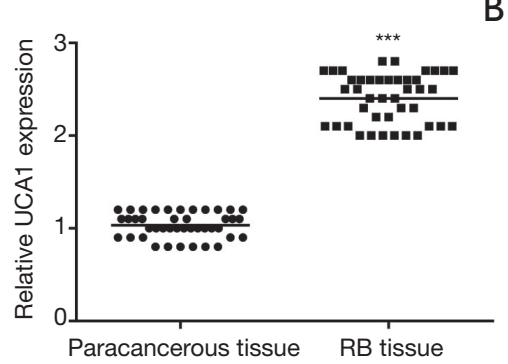

B

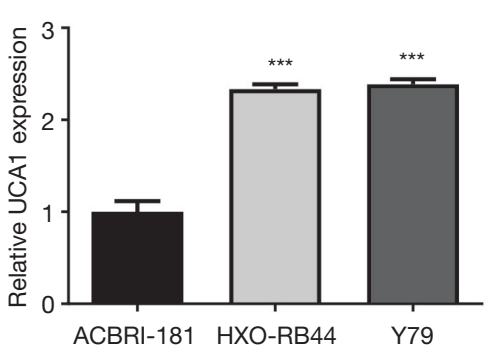

C

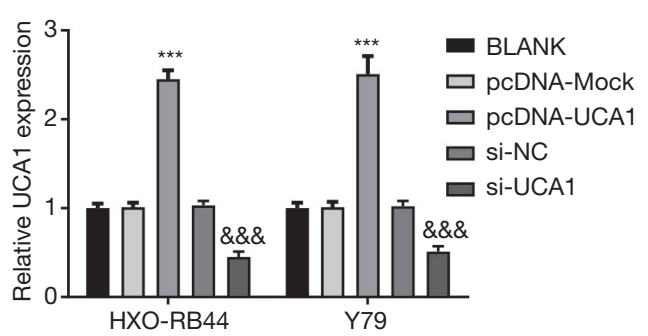

Figure 1 LncRNA-UCA1 expression in both RB tissues and HXO-RB44 and Y79 cells. The expression of lncRNA-UCA1 in RB tissues and adjacent tissue (A), ACBRI-181, HXO-RB44 and Y79 cells (B), and transfected HXO-RB44 and Y79 cells (C). *** $\mathrm{P}<0.01$ vs. paracancerous tissue group (A); ${ }^{* * *}, \mathrm{P}<0.001$ vs. ACBRI-181 group (B); ${ }^{* * *}, \mathrm{P}<0.01$, vs. BLANK and pcDNA-Mock group; ${ }^{\text {*\&\& }}, \mathrm{P}<0.01$, vs. BLANK and siNC group (C). RB, retinoblastoma.

Abcam, USA); survivin, 1:1,000; CDK2, 1:1,000; caspase-3, 1:1,000; p16, 1:1,000; and p21, 1:1,000 (all from Cell Signal, USA)]. Subsequently, the membranes were incubated with a peroxidase-labelled secondary antibody (antirabbit IgG, 1:5,000, I5381MSDS; Sigma, USA) for $1 \mathrm{~h}$. Finally, the protein bands were detected with an enhanced chemiluminescence (ECL) kit (Thermo Fisher).

\section{Statistical analysis}

All statistical analyses were performed using SPSS 22.0 Statistical Software (Chicago, IL, USA). The results are presented as the mean $\pm \mathrm{SD}$. Statistical significance was tested using one-way ANOVA or Student's $t$-test. A P value less than 0.05 was considered to be statistically significant.

\section{Results}

The expression of IncRNA-UCA1 is upregulated in $R B$ and is associated with several clinical characteristics

The qRT-PCR results showed that IncRNA-UCA1 expression levels in $\mathrm{RB}$ samples were markedly higher than those in adjacent non-cancerous tissues $(\mathrm{P}<0.01)$ (Figure 1A). Similarly, lncRNA-UCA1 expression was markedly higher in HXO-RB44 and Y79 cells than in ACBRI-181 cells $(\mathrm{P}<0.01)$ (Figure $1 B)$. These results suggested that lncRNAUCA1 was highly expressed in RB. We also found that IncRNA-UCA1 expression was correlated with tumour size $(\mathrm{P}<0.01)$, optic nerve invasion $(\mathrm{P}<0.01)$, and pathologic grade $(\mathrm{P}<0.01)$ (Table 1). However, IncRNA-UCA1 expression was not significantly correlated with the age or gender of patients with RB ( $>>0.05)$ (Table 1).
LncRNA-UCA1 expression in HXO-RB44 and Y79 cells was significantly higher in the pcDNA-UCA1 group than in the BLANK and pcDNA-Mock groups $(\mathrm{P}<0.01)$ (Figure $1 C$ ). LncRNA-UCA1 expression was markedly decreased in HXO-RB44 and Y79 cells in the si-UCA1 group than in BLANK and si-NC groups $(\mathrm{P}<0.01)$ (Figure 1C), suggesting that the transfections were successful.

\section{LncRNA-UCA1 promotes cell proliferation in HXO-RB44 and Y79 cells}

The results of the CCK-8 assay showed that the proliferation of HXO-RB44 cells was markedly lower in the si-UCA1 group than in the BLANK and si-NC groups at $48 \mathrm{~h}$ $(\mathrm{P}<0.05)$ and at 72 and $96 \mathrm{~h}(\mathrm{P}<0.01)$ (Figure $2 A)$. When compared with the BLANK and pcDNA-Mock groups, the proliferation ability of $\mathrm{Y} 79$ cells was markedly higher in the pcDNA-UCA1 group at $48 \mathrm{~h}(\mathrm{P}<0.05)$ and at 72 and $96 \mathrm{~h}(\mathrm{P}<0.01)$ (Figure $2 A)$. The colony formation assay also confirmed that silencing of lncRNA-UCA1 expression markedly decreased the number of HXO-RB44 cell colonies $(\mathrm{P}<0.01)$ (Figure $2 B$ ), whereas lncRNA-UCA1 overexpression significantly increased the number of $\mathrm{Y} 79$ cell colonies $(\mathrm{P}<0.01)$ (Figure $2 B)$. To further confirm the effect of lncRNA-UCA1 on RB cell proliferation, we also measured the expression of PCNA in both HXO-RB44 and Y79 cells. PCNA expression in HXO-RB44 cells was markedly lower in the si-UCA1 group than in the BLANK and si-NC groups $(\mathrm{P}<0.01)$ (Figure 2C). When compared with the BLANK and pcDNA-Mock groups, PCNA expression in Y79 cells was markedly increased in the pcDNA-UCA1 group $(\mathrm{P}<0.01)$ (Figure 2C). These results indicated that lncRNA-UCA1 promotes the proliferation of $\mathrm{RB}$ cells. 
A

HXO-RB44

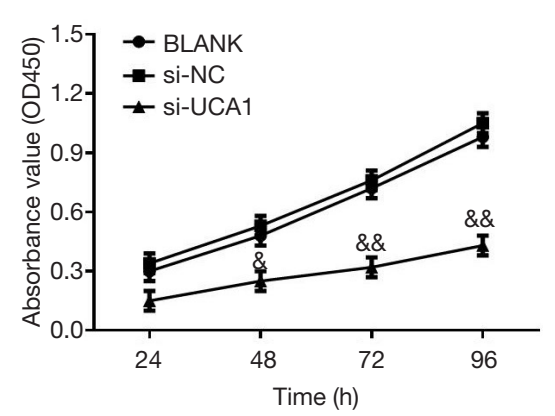

B
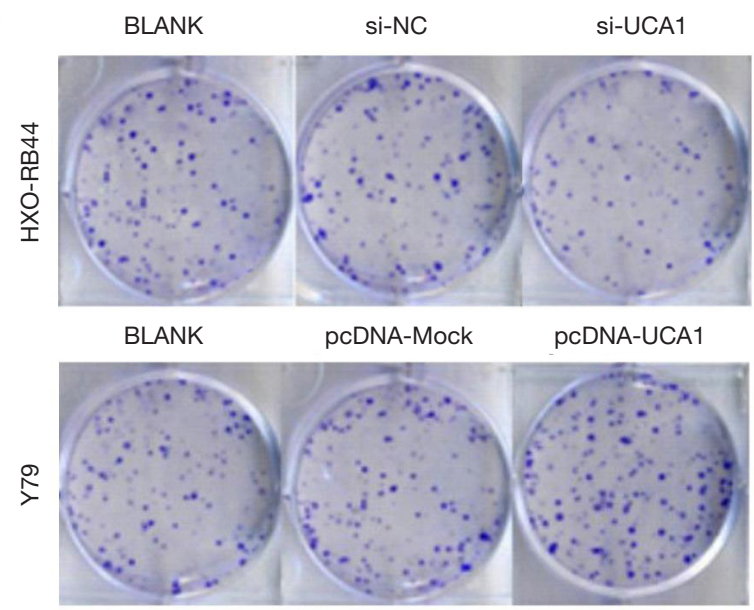

C

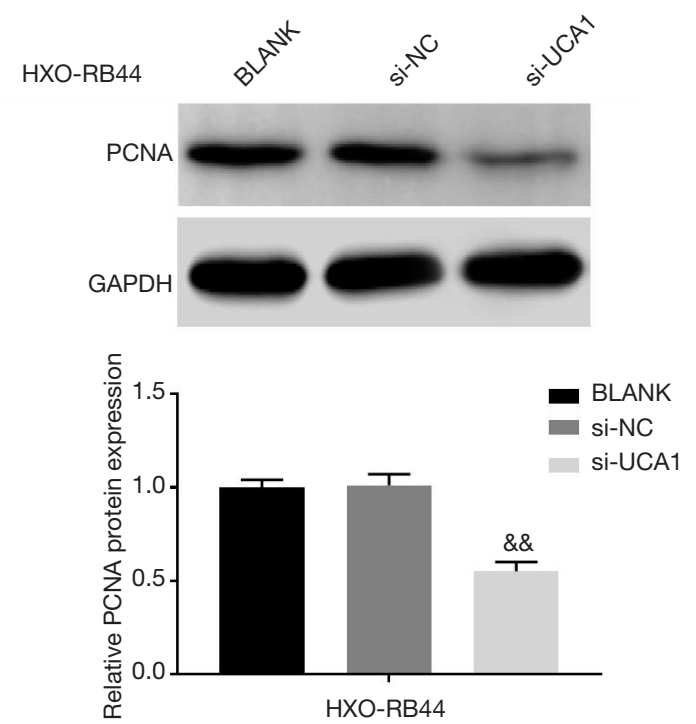

Y79
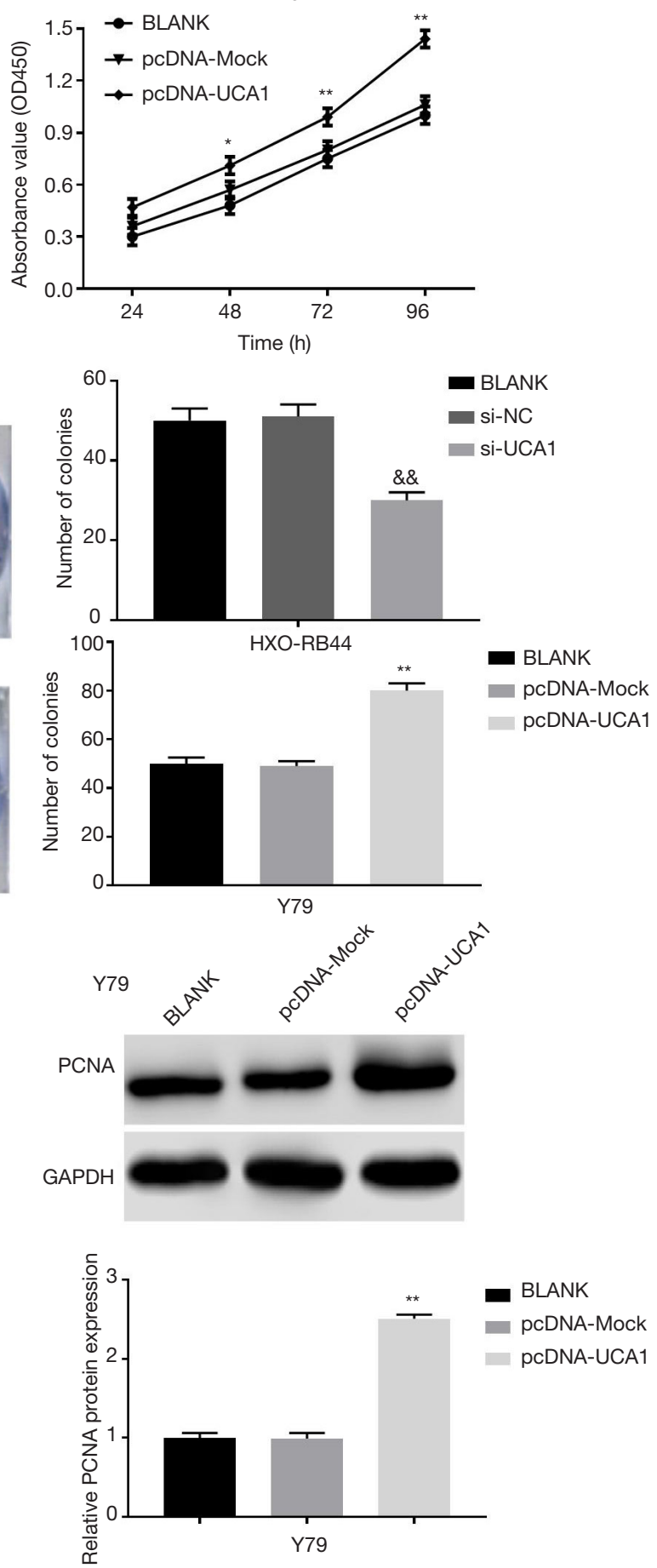

Figure 2 LncRNA-UCA1 promoted cell proliferation in HXO-RB44 and Y79 cells. (A) The cell proliferation of transfected HXO-RB44 and Y79 cells; (B) the number of transfected HXO-RB44 and Y79 cell colonies; (C) the expression levels of PCNA in transfected HXORB44 and Y79 cells. *, $\mathrm{P}<0.05 ;{ }^{* *}, \mathrm{P}<0.01$, vs. BLANK and pcDNA-Mock group; ${ }^{\star}, \mathrm{P}<0.05 ;{ }^{* \star \star}, \mathrm{P}<0.01$, vs. BLANK and si-NC group. 
A

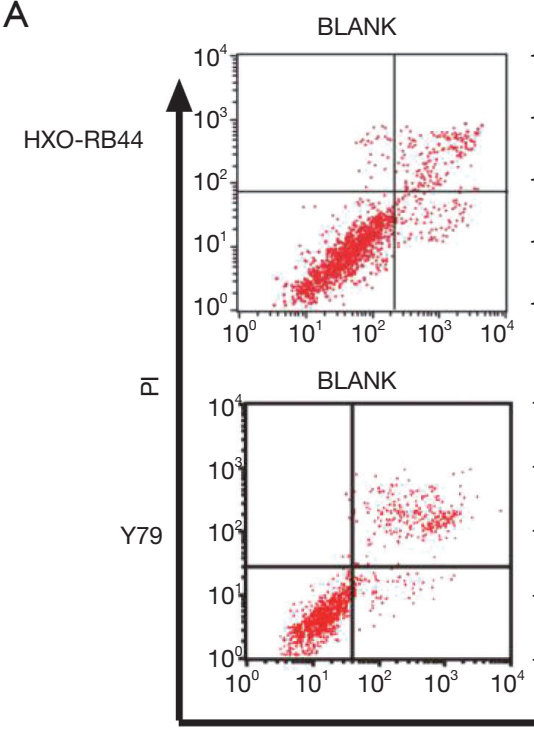

B

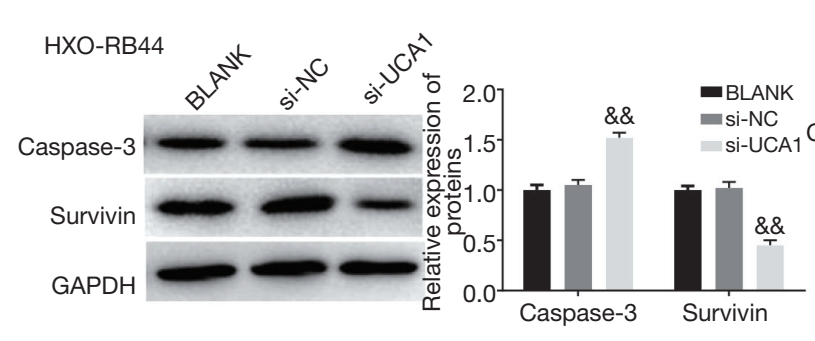

si-NC
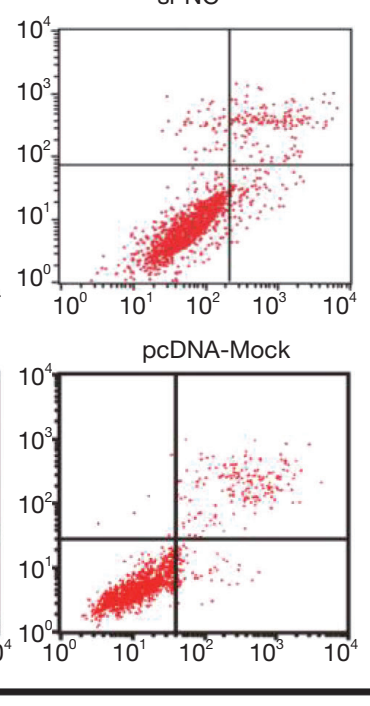
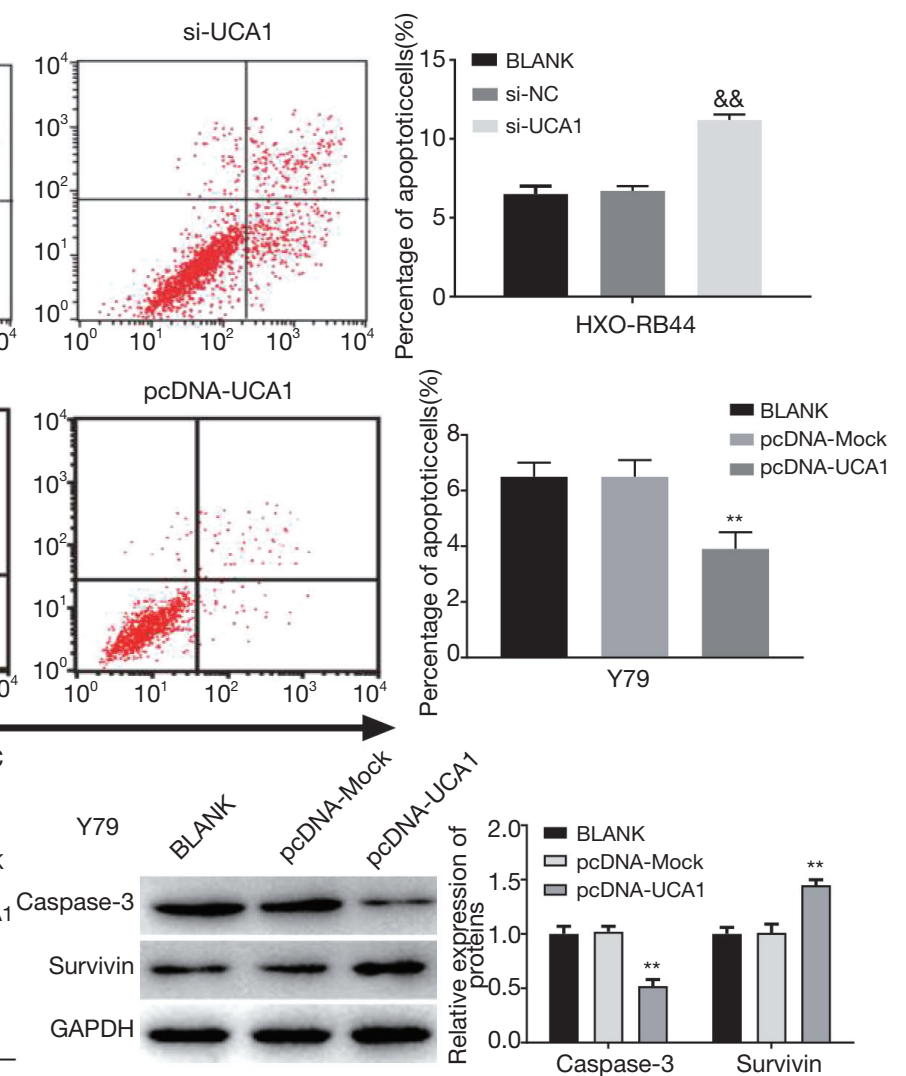

Figure 3 LncRNA-UCA1 inhibited cell apoptosis in HXO-RB44 and Y79 cells. (A) The apoptosis of transfected HXO-RB44 and Y79 cells; (B) the expression levels of caspase- 3 and survivin in transfected HXO-RB44 and Y79 cells. ${ }^{* *}, \mathrm{P}<0.01$, $v$ s. BLANK and pcDNA-Mock group; ${ }^{\text {\&\&, }}, \mathrm{P}<0.01, v s$. BLANK and si-NC group.

\section{LncRNA-UCA1 inbibits apoptosis in HXO-RB44 and Y79 cells}

As shown in Figure $3 A$, silencing of lncRNA-UCA1 expression dramatically increased apoptosis in HXORB44 cells $(\mathrm{P}<0.01)$ (Figure $3 A)$, whereas lncRNA-UCA1 overexpression significantly decreased apoptosis in Y79 cells $(\mathrm{P}<0.01)$. To further confirm the effect of $\operatorname{lncRNA}-\mathrm{UCA} 1$ on $\mathrm{RB}$ cell apoptosis, we detected the expression levels of caspase-3 and survivin in HXO-RB44 and Y79 cells. Silencing of lncRNA-UCA1 markedly increased caspase-3 expression and decreased survivin expression in HXO-RB44 cells $(\mathrm{P}<0.01)$ (Figure $3 B)$. In contrast, IncRNA-UCA1 overexpression markedly decreased caspase- 3 expression and increased survivin expression in $\mathrm{Y} 79$ cells $(\mathrm{P}<0.01)$ (Figure $3 B$ ). These results suggested that lncRNA-UCA1 can inhibit apoptosis in RB cells.

\section{LncRNA-UCA1 promotes cell cycle progression in HXO- RB44 and $Y 79$ cells}

As shown in Figure 4A, silencing of lncRNA-UCA1 in $\mathrm{HXO}-\mathrm{RB} 44$ cells dramatically increased the percentage of cells in $\mathrm{G} 0 / \mathrm{G} 1$ phase $(\mathrm{P}<0.01)$ and notably reduced the percentage of cells in $\mathrm{S}(\mathrm{P}<0.01)$ and $\mathrm{G} 2 / \mathrm{M}$ phases $(\mathrm{P}<0.01)$. In contrast, lncRNA-UCA1 overexpression in Y79 cells dramatically decreased the percentage of cells in G0/G1 phase $(\mathrm{P}<0.01)$ and markedly increased the percentage of cells in $\mathrm{S}(\mathrm{P}<0.01)$ and $\mathrm{G} 2 / \mathrm{M}$ phases $(\mathrm{P}<0.01)$. Additionally, silencing of lncRNA-UCA1 significantly increased p16 and p21 expression levels and decreased CDK2 expression levels in HXO-RB44 cells $(\mathrm{P}<0.01)$ (Figure 4B), and overexpression of lncRNA-UCA1 had the opposite effects (Figure $4 B$ ). These results indicated that lncRNA-UCA1 could promote cell cycle progression in RB cells. 

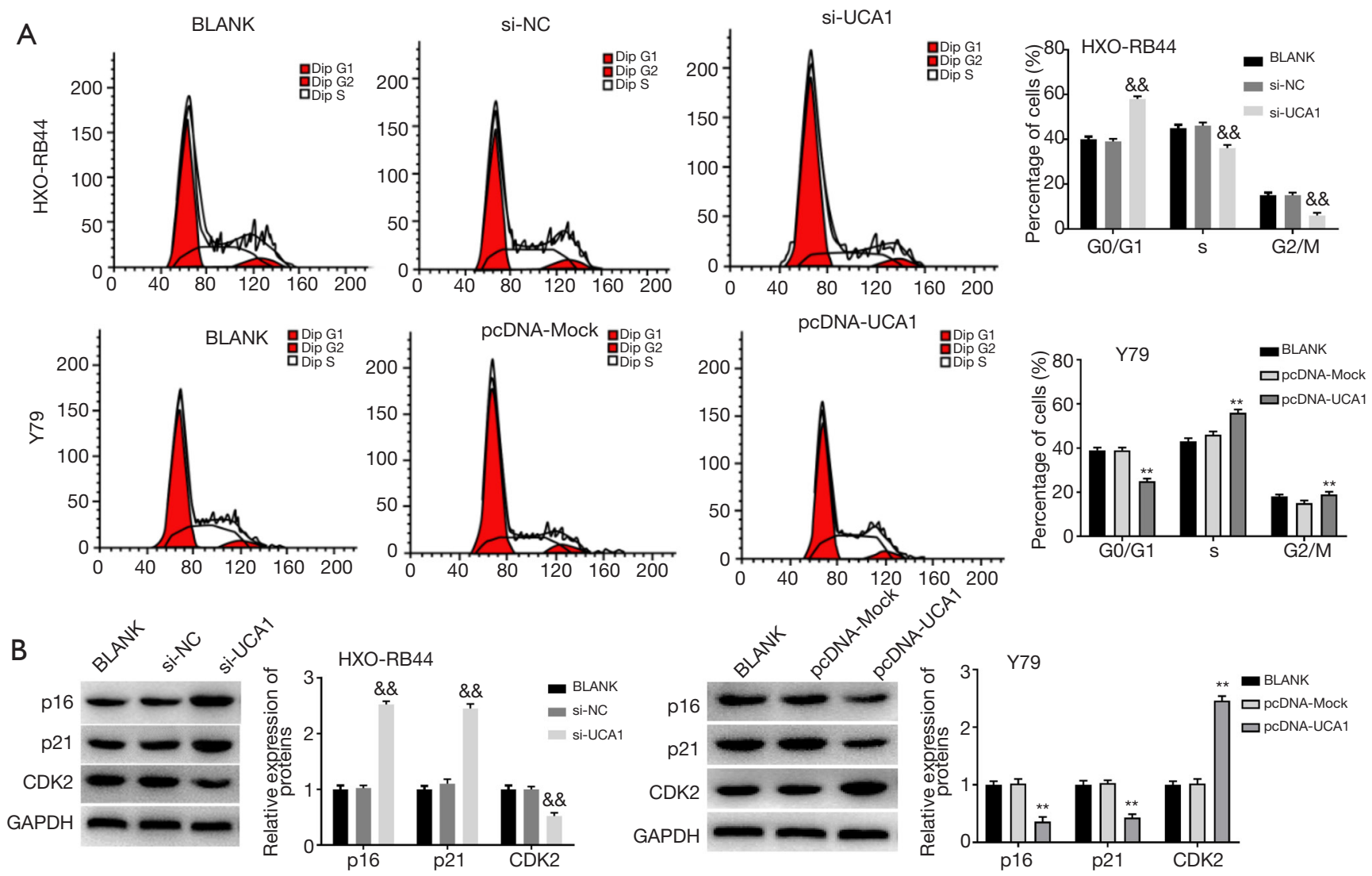

Figure 4 LncRNA-UCA1 promoted cell cycle procession in HXO-RB44 and Y79 cells. (A) The cell cycle distribution in transfected HXORB44 and Y79 cells; (B) the expression levels of p16, p21 and CDK2 in transfected HXO-RB44 and Y79 cells. **, P<0.01, vs. BLANK and pcDNA-Mock group; ${ }^{\& \&}, \mathrm{P}<0.01$, vs. BLANK and si-NC group.

\section{LncRNA-UCA1 activates the PI3K/Akt signalling pathway in HXO-RB44 and Y79 cells}

The expression levels of p-PI3K, p-Akt, and S6K in HXORB44 cells in the si-UCA1 group were markedly lower than those in the si-UCA1 group and were markedly higher in the pcDNA-UCA1 group than in the BLANK and pcDNAMock groups $(\mathrm{P}<0.01)$ (Figure 5), suggesting that lncRNAUCA1 could activate the PI3K/Akt signalling pathway in HXO-RB44 and Y79 cells.

\section{LncRNA-UCA1 promotes cell proliferation and cell cycle progression and inbibits apoptosis in $R B$ by activating the PI3K/Akt signalling patbway}

To further confirm that lncRNA-UCA1 exerts its effects on $\mathrm{RB}$ through activation of the PI3K/Akt signalling pathway, we performed a variety of experiments in Y79 cells. The results of CCK-8 and colony formation assays showed that Y79 cell proliferation was markedly promoted in the pcDNA-UCA1 group when compared with that in the BLANK and pcDNA-Mock groups $(\mathrm{P}<0.01)$ (Figure 6A,B). Treatment of cells with LY294002 markedly reversed the effects of IncRNA-UCA1 on cell proliferation $(\mathrm{P}<0.01)$ (Figure 6A,B). As shown in Figure 6C, apoptosis in Y79 cells was significantly lower in the pcDNA-UCA1 group than in the BLANK and pcDNA-Mock groups $(\mathrm{P}<0.01)$. When compared with the pcDNA-UCA1 group, apoptosis in Y79 cells was markedly higher in the pcDNAUCA1 + LY294002 group $(\mathrm{P}<0.01)$. In addition, we also confirmed that overexpression of IncRNA-UCA1 in Y79 cells dramatically decreased the percentage of cells in G0/G1 phase $(\mathrm{P}<0.01)$ and markedly increased percentage of cells in $\mathrm{S}(\mathrm{P}<0.01)$ and $\mathrm{G} 2 / \mathrm{M}$ phases $(\mathrm{P}<0.01)$ (Figure $6 D)$. Treatment with LY294002 significantly reversed the effects 
A

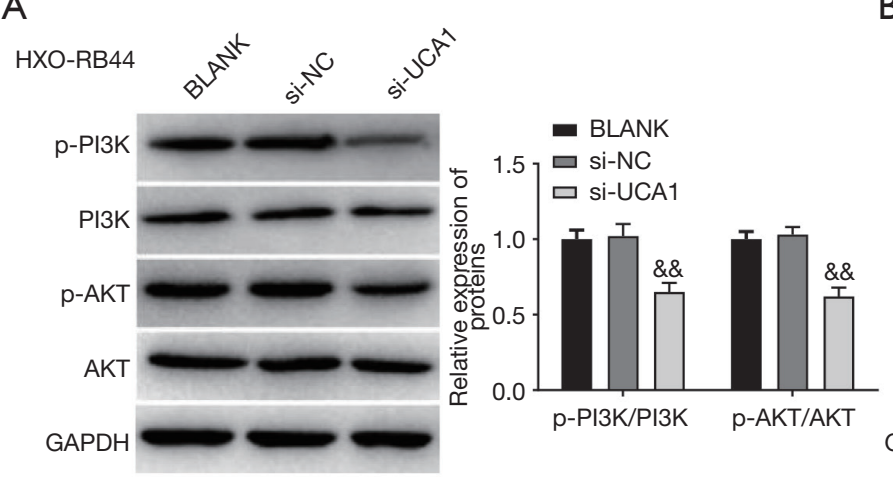

B

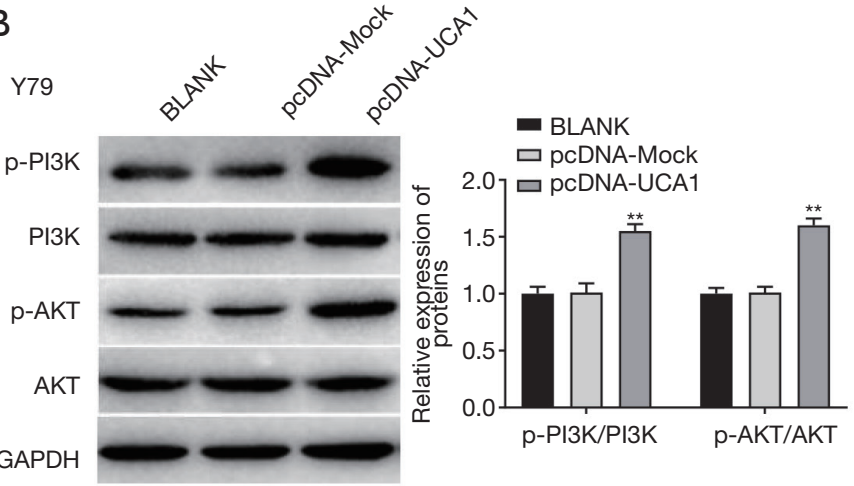

Figure 5 LncRNA-UCA1 activated PI3K/Akt signaling pathway in HXO-RB44 and Y79 cells. ** ${ }^{*}<0.01, v s$. BLANK and pcDNA-Mock group; ${ }^{\& \&}, \mathrm{P}<0.01, v s$. BLANK and si-NC group.

of lncRNA-UCA1 on the cell cycle $(\mathrm{P}<0.01)$ (Figure $6 D)$. The Western blot results showed that caspase-3, p16, and p21 levels in the pcDNA-UCA1 group were markedly lower than those in the BLANK and pcDNA-Mock groups $(\mathrm{P}<0.01)$, while the levels of survivin and CDK2 were significantly higher $(\mathrm{P}<0.01)$ (Figure $6 E, F)$. When compared with the pcDNA-UCA1 group, the expression levels of caspase-3, p16, and p21 in Y79 cells were markedly higher in the pcDNA-UCA1 + LY294002 group $(\mathrm{P}<0.01)$, while the expression levels of survivin and CDK2 were significantly lower $(\mathrm{P}<0.01)$ (Figure $6 E, F)$. These results indicated that IncRNA-UCA1 could promote cell proliferation and cell cycle progression and inhibit apoptosis in RB by activating the PI3K/Akt pathway.

\section{Discussion}

$\mathrm{RB}$ is the most common intraocular malignancy, and it is diagnosed in approximately 8,000 children worldwide each year (18). In developing countries, the survival rate of RB patients is $<70 \%$ (19). Thus, new, effective molecular targets are needed for the treatment of RB. In this study, we confirmed that IncRNA-UCA1 could facilitate cell proliferation and inhibit apoptosis in RB by activating the PI3K/Akt pathway.

Accumulating evidence suggests that many lncRNAs play carcinogenic or anticancer roles in various malignant tumours, such as RB $(20,21)$. In addition, many lncRNAs have been reported to be abnormally expressed in tumours. Previous studies have confirmed that the expression of IncRNA-UCA1 is upregulated in gastric cancer $(16,22)$ and lung cancer (23), and Sun et al. (24) reported that lncRNA-
UCA1 is highly expressed in laryngeal squamous cell carcinoma. In the present study, we assessed the expression of lncRNA-UCA1 in both RB tissues and HXO-RB44 and $\mathrm{Y79}$ RB cells and found that it was highly expressed in both RB tissues and both RB cell lines. Furthermore, we also found that lncRNA-UCA1 expression was associated with some clinical characteristics of RB patients, such as tumour size, optic nerve invasion, and pathologic grade. Accumulating evidence has confirmed that some lncRNAs can influence cell proliferation, migration, apoptosis, and the cell cycle in various malignancies (25). For example, IncRNA-CCAT1 promoted cell proliferation, migration, and invasion and suppressed apoptosis in RB cells by modulating miR-218-5p (26); Li et al. (27) reported that lncRNA-00152 promoted cell proliferation and migration and suppressed apoptosis in RB; an lncRNA-H19 suppressed the proliferation of RB cells and promoted cell cycle arrest and apoptosis by counteracting the miR-1792 cluster (28). In this study, IncRNA-UCA1 promoted cell proliferation and cell cycle progression and inhibited apoptosis in RB, indicating that IncRNA-UCA1 may be useful as a prognostic biomarker for RB.

Accumulating research suggests that the PI3K/Akt signalling pathway affects various biological functions in the development of progression of malignancies, including cell proliferation, migration, invasion, apoptosis, and the cell cycle (29). S6K is a downstream target of the PI3K/ Akt pathway. When the PI3K/Akt pathway is activated, S6K is activated, which regulates various crucial cellular activities (17). Previous findings indicated that the effects of lncRNAs on various cellular functions in many cancers involves modulation of the PI3K/Akt pathway. For instance, 
A

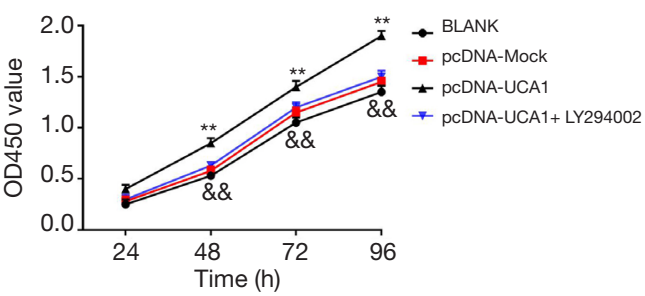

C
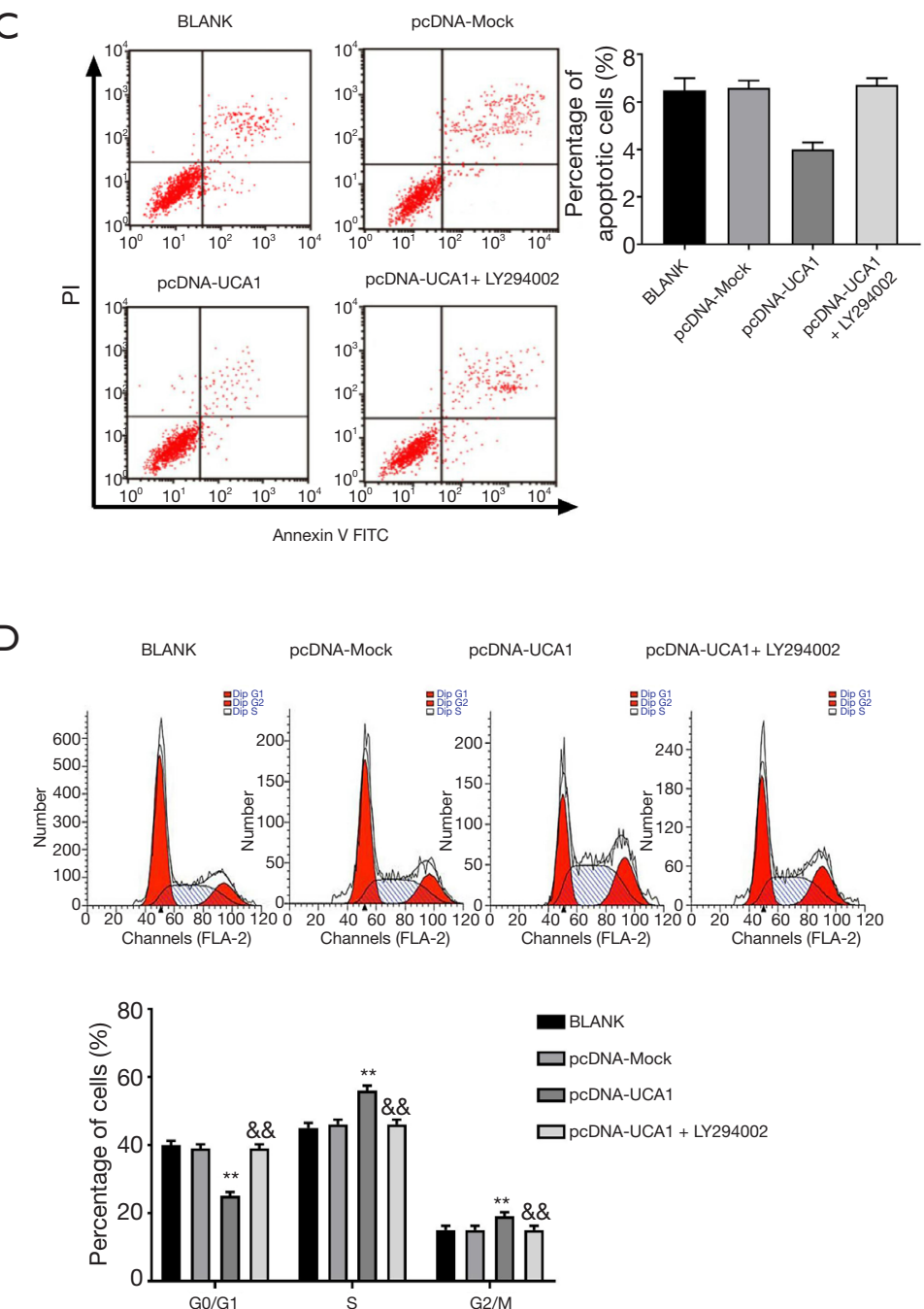

$\mathrm{E}$

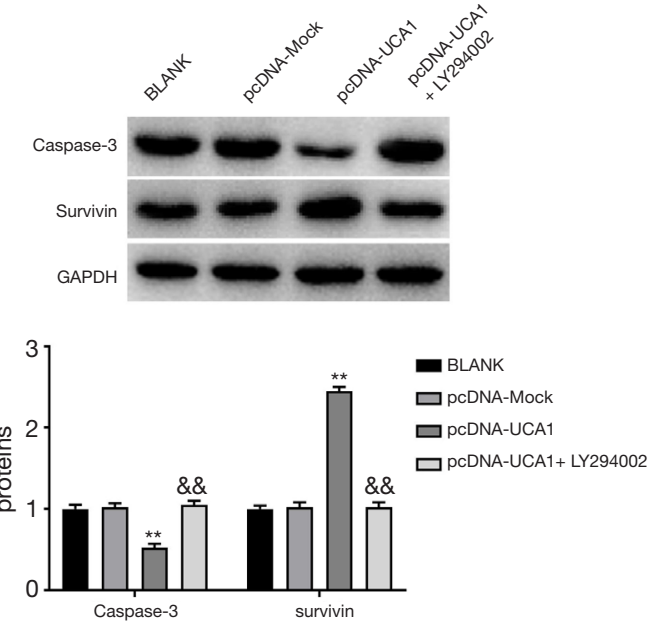

$\mathrm{F}$
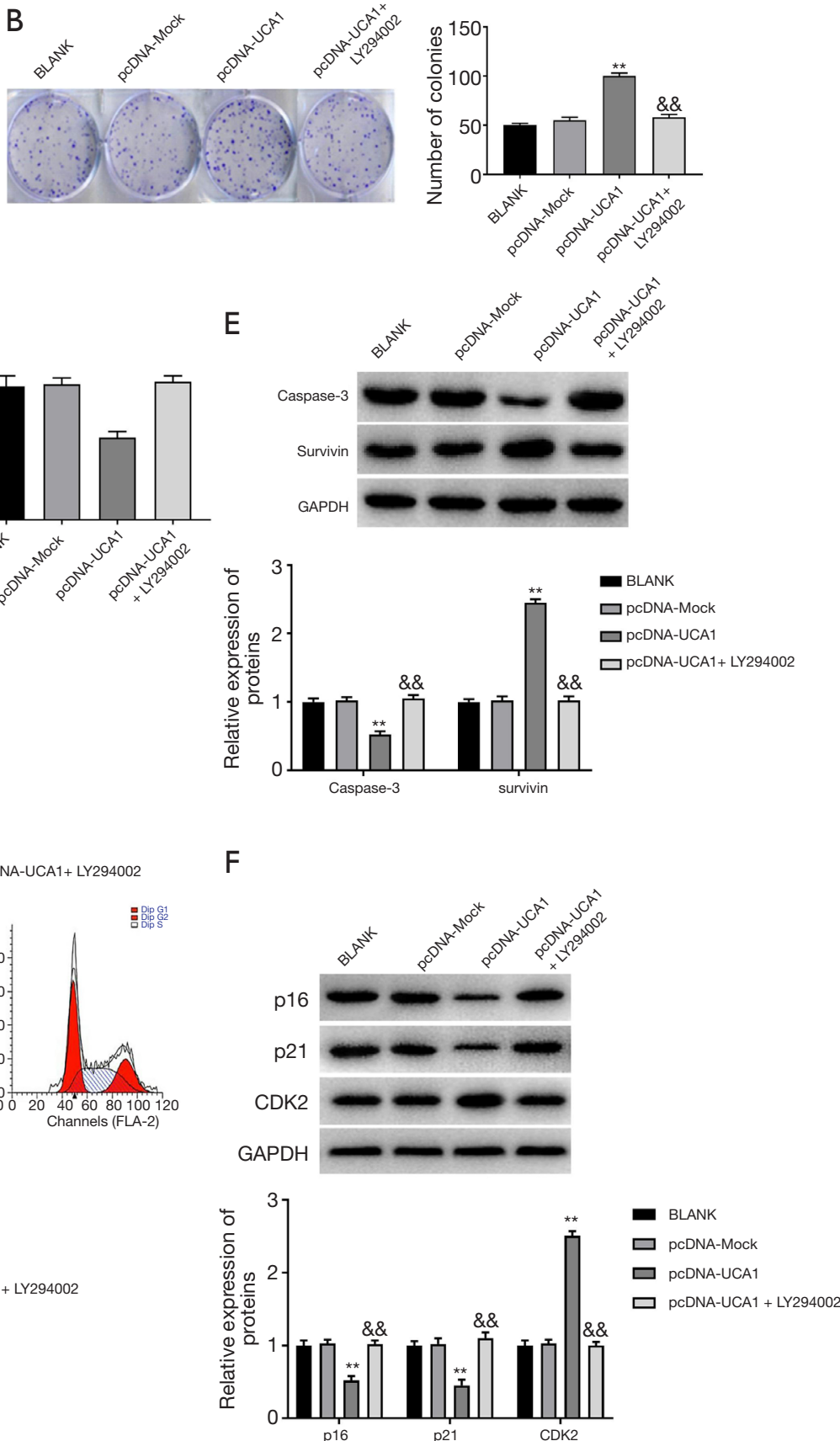

Figure 6 LncRNA-UCA1 promoted cell proliferation and cell cycle procession and inhibited apoptosis in RB by activating PI3K/Akt pathway. (A) The cell proliferation of transfected Y79 cells; (B) the number of transfected Y79 cell colonies; (C) the apoptosis of transfected Y79 cells; (D) the cell cycle distribution in transfected Y79 cells; (E) the expression levels of Caspase-3 and survivin in transfected Y79 cells; (F) the expression levels of p16, p21 and CDK2 in transfected Y79 cells. ${ }^{* *}, \mathrm{P}<0.01, v s$. BLANK and pcDNA-Mock group; ${ }^{\text {\&\& }}, \mathrm{P}<0.01, v s$. pcDNA-UCA1 group. RB, retinoblastoma. 
IncRNA-UCA1 has been reported to regulate cell cycle progression through the PI3K signalling pathway in bladder carcinoma cells (30). The results of a study of $\mathrm{Li}$ et al. (17) indicated that IncRNA-UCA1 significantly promotes cell proliferation and suppresses apoptosis by activating the PI3K-Akt-mTOR signalling pathway in gastric cancer. Ma et al. (16) reported that lncRNA-UCA1 could promote metastasis in osteosarcoma through activation of the PI3K/ Akt signalling pathway. In the present study, silencing of lncRNA-UCA1 significantly decreased phosphorylation of PI3K and Akt and the expression of S6k in HXO-RB44 cells, while lncRNA-UCA1 overexpression dramatically promoted phosphorylation of PI3K and expression of Akt and S6k in Y79 cells, suggesting that lncRNA-UCA1 could activate the PI3K/Akt signalling pathway in RB cells. In addition, we also confirmed that LY294002 reversed the effects of lncRNA-UCA1 on proliferation, apoptosis, and cell cycle progression in RB cells. These results indicate that IncRNA-UCA1 could promote cell proliferation and cell cycle progression and inhibit apoptosis in RB by activating the PI3K/Akt pathway.

\section{Conclusions}

In conclusion, our study confirmed that lncRNA-UCA1 expression was dramatically upregulated in both RB tissues and HXO-RB44 and Y79 RB cells. In addition, we found that lncRNA-UCA1 promoted cell proliferation and cell cycle progression and inhibited apoptosis by activating the PI3K/Akt pathway in RB. Our research provides evidence for a novel regulatory mechanism involving lncRNA-UCA1 in $\mathrm{RB}$ and suggests a potential new target for RB treatment.

\section{Acknowledgments}

Funding: None.

\section{Footnote}

Conflicts of Interest: Both authors have completed the ICMJE uniform disclosure form (available at http://dx.doi. org/10.21037/tcr.2019.12.47). The authors have no conflicts of interest to declare.

Ethical Statement: The authors are accountable for all aspects of the work in ensuring that questions related to the accuracy or integrity of any part of the work are appropriately investigated and resolved. The study was conducted in accordance with the Declaration of Helsinki (as revised in 2013). The study was approved by the Ethics Committee of our hospital (No. 2017_354). All patients or their guardians provided written informed consent.

Open Access Statement: This is an Open Access article distributed in accordance with the Creative Commons Attribution-NonCommercial-NoDerivs 4.0 International License (CC BY-NC-ND 4.0), which permits the noncommercial replication and distribution of the article with the strict proviso that no changes or edits are made and the original work is properly cited (including links to both the formal publication through the relevant DOI and the license). See: https://creativecommons.org/licenses/by-nc-nd/4.0/.

\section{References}

1. Rao R, Honavar SG. Retinoblastoma. Indian J Pediatr 2017;84:937-44.

2. Dimaras H, Dimba EA, Gallie BL. Challenging the global retinoblastoma survival disparity through a collaborative research effort. Br J Ophthalmol 2010;94:1415-6.

3. Lukamba RM, Yao JA, Kabesha TA, et al. Retinoblastoma in Sub-Saharan Africa: Case Studies of the Republic of Cote d'Ivoire and the Democratic Republic of the Congo. J Glob Oncol 2018;4:1-8.

4. Kivelä T. The epidemiological challenge of the most frequent eye cancer: retinoblastoma, an issue of birth and death. Br J Ophthalmol 2009;93:1129-31.

5. Peng WX, Koirala P, Mo YY. LncRNA-mediated regulation of cell signaling in cancer. Oncogene 2017;36:5661-7.

6. Wang L, Yang D, Tian R, et al. NEAT1 promotes retinoblastoma progression via modulating miR-124. J Cell Biochem 2019;120:15585-93.

7. Chen L, Dzakah EE, Shan G. Targetable long non-coding RNAs in cancer treatments. Cancer Lett 2018;418:119-24.

8. Hu C, Liu S, Han M, et al. Knockdown of lncRNA XIST inhibits retinoblastoma progression by modulating the miR-124/STAT3 axis. Biomed Pharmacother 2018;107:547-54.

9. Shang W, Yang Y, Zhang J, et al. Long noncoding RNA BDNF-AS is a potential biomarker and regulates cancer development in human retinoblastoma. Biochem Biophys Res Commun 2018;497:1142-8.

10. Xu C, Hu C, Wang Y, et al. Long noncoding RNA SNHG16 promotes human retinoblastoma progression via sponging miR-140-5p. Biomed Pharmacother 
2019;117:109153

11. Dong C, Liu S, Lv Y, et al. Long non-coding RNA HOTAIR regulates proliferation and invasion via activating Notch signalling pathway in retinoblastoma. J Biosci 2016;41:677-87.

12. Qi D, Wang M, Yu F. Knockdown of lncRNA-H19 inhibits cell viability, migration and invasion while promotes apoptosis via microRNA-143/RUNX2 axis in retinoblastoma. Biomed Pharmacother 2019;109:798-805.

13. Zhang Y, Kwok-Shing Ng P, Kucherlapati M, et al. A Pan-Cancer Proteogenomic Atlas of PI3K/AKT/mTOR Pathway Alterations. Cancer Cell 2017;31:820-832.e3.

14. Zeng L, Liao Q, Zou Z, et al. Long Non-Coding RNA XLOC_006753 Promotes the Development of Multidrug Resistance in Gastric Cancer Cells Through the PI3K/ AKT/mTOR Signaling Pathway. Cell Physiol Biochem 2018;51:1221-36.

15. Li D, Ji H, Niu X, et al. Tumor-associated macrophages secrete CCL2 and induce tamoxifen resistance by activating $\mathrm{PI} 3 \mathrm{~K} / \mathrm{Akt} / \mathrm{m}$ TOR in breast cancer. Cancer Sci 2019;11:14230.

16. Ma H, Su R, Feng H, et al. Long noncoding RNA UCA1 promotes osteosarcoma metastasis through CREB1-mediated epithelial-mesenchymal transition and activating PI3K/AKT/mTOR pathway. J Bone Oncol 2019;16:100228.

17. Li C, Liang G, Yang S, et al. Dysregulated lncRNA-UCA1 contributes to the progression of gastric cancer through regulation of the PI3K-Akt-mTOR signaling pathway. Oncotarget 2017;8:93476-91.

18. Soliman SE, Racher H, Zhang C, et al. Genetics and Molecular Diagnostics in Retinoblastoma--An Update. Asia Pac J Ophthalmol (Phila) 2017;6:197-207.

19. Fabian ID, Onadim Z, Karaa E, et al. The management of retinoblastoma. Oncogene 2018;37:1551-60.

20. Shang Y. LncRNA THOR acts as a retinoblastoma promoter through enhancing the combination of c-myc mRNA and IGF2BP1 protein. Biomed Pharmacother

Cite this article as: Yuan Z, Li Z. Long noncoding RNA UCA1 facilitates cell proliferation and inhibits apoptosis in retinoblastoma by activating the PI3K/Akt pathway. Transl Cancer Res 2020;9(2):1012-1022. doi: 10.21037/tcr.2019.12.47
2018;106:1243-9.

21. Lyu X, Ma Y, Wu F, et al. LncRNA NKILA Inhibits Retinoblastoma by Downregulating lncRNA XIST. Curr Eye Res 2019;44:975-9.

22. He X, Wang J, Chen J, et al. IncRNA UCA1 Predicts a Poor Prognosis and Regulates Cell Proliferation and Migration by Repressing p21 and SPRY1 Expression in GC. Mol Ther Nucleic Acids 2019;18:605-16.

23. Nie W, Ge HJ, Yang XQ, et al. LncRNA- UCA1 exerts oncogenic functions in non-small cell lung cancer by targeting miR-193a-3p. Cancer Letters 2016;371:99-106.

24. Sun S, Gong C, Yuan K. LncRNA UCA1 promotes cell proliferation, invasion and migration of laryngeal squamous cell carcinoma cells by activating Wnt/betacatenin signaling pathway. Exp Ther Med 2019;17:1182-9.

25. Gao D, Lv AE, Li HP, et al. LncRNA MALAT-1 Elevates HMGB1 to Promote Autophagy Resulting in Inhibition of Tumor Cell Apoptosis in Multiple Myeloma. J Cell Biochem 2017;118:3341-8.

26. Zhang H, Zhong J, Bian Z, et al. Long non-coding RNA CCAT1 promotes human retinoblastoma SO-RB50 and Y79 cells through negative regulation of miR-218-5p. Biomed Pharmacother 2017;87:683-91.

27. Li S, Wen D, Che S, et al. Knockdown of long noncoding RNA 00152 (LINC00152) inhibits human retinoblastoma progression. Onco Targets Ther 2018;11:3215-23.

28. Zhang A, Shang W, Nie Q, et al. Long non-coding RNA H19 suppresses retinoblastoma progression via counteracting miR-17-92 cluster. J Cell Biochem 2018;119:3497-509.

29. Chen S, Fisher RC, Signs S, et al. Inhibition of PI3K/ Akt/mTOR signaling in PI3KR2-overexpressing colon cancer stem cells reduces tumor growth due to apoptosis. Oncotarget 2016;8:50476-88.

30. Yang C, Li X, Wang Y, et al. Long non-coding RNA UCA1 regulated cell cycle distribution via CREB through PI3-K dependent pathway in bladder carcinoma cells. Gene 2012;496:8-16. 